\title{
Risk of coronary arterial lesions in immunoglobulin resistant Kawasaki disease
}

\author{
Hirotaro Ogino $^{1}$, Kazunari Kaneko ${ }^{1 *}$, Takamichi Uchiyama ${ }^{1}$, Ken Yoshimura ${ }^{1}$, Masayuki Teraguchi ${ }^{1}$, \\ Yosikazu Nakamura ${ }^{2}$ \\ ${ }^{1}$ Department of Pediatrics, Kansai Medical University, Osaka, Japan \\ ${ }^{2}$ Department of Public Health, Jichi Medical University, Tochigi, Japan \\ Email: "kanekok@hirakata.kmu.ac.jp
}

Received 7 October 2011; revised 19 November 2011; accepted 8 December 2011

\begin{abstract}
Objective: High-dose intravenous immunoglobulin (IVIG) decreases the risk for development of coronary arterial lesions (CAL) in Kawasaki disease (KD) while patients resistant to IVIG have higher risk. This study is aimed to investigate an additional therapy affects the risk for CAL in IVIG resistant KD. Design: The information from 11,510 children with KD was collected by the nationwide survey (2003-2004) in Japan. The secondary therapeutic strategies for IVIG resistant KD were an additional IVIG dose, steroids or both. The incidence of CAL was compared among 4 groups: G1, children responded to initial IVIG; G2, IVIG resistant patients receiving an additional IVIG dose; G3, IVIG resistant patients receiving additional steroids; G4, IVIG resistant patients receiving an additional IVIG dose plus steroids. CAL was assessed at 30th day of illness using ultrasound echocardiography. Results: 2229 patients (19.4\%) were resistant to initial IVIG. Incidence of CAL was significantly lower in children responding to initial IVIG than in IVIG resistant patients $(1.9 \%$ and $11.0 \%$, respectively; odd's ratio 6.3). The incidences of CAL in an each additional therapy group were as follows: G2: 6.7\%, G3: 9.7\%, G4: $22.2 \%$. The risk for CAL was significantly higher in G4 as compared with G2 and G3. Giant aneurysmal formation (GA) was more frequently developed in G3 and G4 consisting of the patients receiving steroids (G1: $0.03 \%$, G2: $0.63 \%$, G3: $4.3 \%$, G4: $3.7 \%$ ). Conclusion: The incidence of CAL was considerably high in IVIG resistant KD and an additional therapy by steroids may increase the risk for GA.
\end{abstract}

Keywords: Kawasaki Disease; Immunoglobulin Resistance; Additional Treatment; Coronary Arterial

${ }^{*}$ Corresponding author.
Lesions; Steroids

\section{INTRODUCTION}

Kawasaki disease (KD) is an acute febrile illness characterized by fever persisting 5 days or more, bilateral conjunctival congestion, changes of lips and oral cavity (reddening of lips, strawberry tongue, diffuse injection of oral and pharyngeal mucosa), polymorphous exanthema, changes of peripheral extremities (initial stage: reddening of palms and soles, indurative edema, convalescent stage: membranous desquamation from fingertips) and acute nonpurulent cervical lymphadenopathy. This disease is an acute systemic vasculitis of unknown etiology that affects primarily small- and medium-sized arteries, particularly the coronary arteries. High-dose intravenous immunoglobulin therapy (IVIG) has decreased the risk for development of coronary arterial lesions (CAL) in $\mathrm{KD}$ [1-3] while approximately $10 \%$ to $15 \%$ of patients showing resistance to initial IVIG have higher risk for CAL [3-8].

The purpose of this study is to determine the risk for CAL in IVIG resistant KD and to investigate whether additional therapy might affect its risk based on the nationwide survey (2003-2004) in Japan.

\section{METHODS}

\subsection{Subjects}

The 18th nationwide survey on KD in Japan was conducted on January 2005 and included patients who visited hospital from January 1, 2003 through December 31, 2004. All pediatric hospitals and general hospitals having a pediatric ward with 100 or more beds were included in the survey. Pediatricians in an each selected hospital were asked to complete a questionnaire for each case of $\mathrm{KD}$ diagnosed over the two-year period. This survey protocol and the questionnaire were reviewed and ap- 
proved by the institutional review board at Jichi Medical University.

Completed questionnaires were returned by 1618 (70.1\%) of 2308 hospitals contacted. A total of 19,138 patients diagnosed as having KD by physicians were reported. Among them, 16,413 (85.8\%) patients received initial IVIG. The information from 11,510 children with KD treated by IVIG $(2 \mathrm{~g} / \mathrm{kg}$ single infusion or $1 \mathrm{~g} / \mathrm{kg}$ for 2 days) within 9 days of the illness was available. In addition, information in regard to the secondary treatment for patients who did not respond to initial IVIG was collected. IVIG resistant KD patient was defined as the child receiving secondary treatment after initial IVIG administration though failure to respond to initial IVIG is generally defined as persistent or recrudescent fever after 36 hours from completion of the initial IVIG infusion [9]. This is because of its nature of retrospective and multicenter survey. Although the secondary treatments for IVIG resistant patients varied considerably, consisting of additional IVIG dose $[4,7,8]$, steroids therapy [10], ulinastatin therapy [11], or plasma exchange therapy [12], minority of patients who received secondary treatment other than IVIG or steroids, such as ulinastatin therapy or plasma exchange therapy, were excluded from the further analysis. CAL was assessed repeatedly within 30th day of illness and finally at the 30th day of illness using ultrasound echocardiography according to the guideline of Japan Kawasaki Disease Research Committee (1984) [13]: coronary arterial dilatation was defined as more than $3 \mathrm{~mm}$ in diameter for a child younger than 5 years old or more than $4 \mathrm{~mm}$ for those 5 years old and above. The patients were considered to be complicated by CAL if they have: 1) dilatation (coronary arterial dilatation detected even at the 30th day, 2) aneurysm: coronary arterial diameter of $4-8 \mathrm{~mm}$ at the 30th day, and 3) giant aneurysm (GA): aneurysmal change of coronary arteries ( $\geq 8 \mathrm{~mm}$ in diameter) at the 30th day.

\subsection{Statistics}

Statistical analysis was performed by chi-square test using SPSS 16.0. P value less than 0.05 was considered significant.

\section{RESULTS}

\subsection{Rate of Initial IVIG Resistant KD Patients and Additional Treatment for Them}

Among 11,510 patients, 9281 responded to initial IVIG while 2229 patients (19.4\%) were resistant to initial IVIG and received additional therapy. These subjects were classified into four groups based on the response to initial IVIG and the subsequent therapeutic strategies: Group 1 (G1), children responded to initial IVIG ( $n=$ 9281); Group 2 (G2), IVIG resistant patients receiving an additional IVIG dose ( $\mathrm{n}=1108)$; Group 3 (G3) IVIG resistant patients given steroids $(\mathrm{n}=93)$; Group 4 (G4), IVIG resistant patients receiving an additional IVIG plus steroids $(\mathrm{n}=135)$.

The remaining 893 patients received secondary treatment other than IVIG or steroids, such as ulinastatin (319 cases) or plasma exchange therapy (total of 14 cases).

\subsection{Incidence of CAL in Relation to Response to Initial IVIG}

Table 1 summarizes the incidence of CAL in the 4 groups. The incidence was significantly lower in children who responded to initial IVIG $(\mathrm{G} 1, \mathrm{n}=9281$; CAL: $1.87 \%$ ) than that in IVIG resistant patients (G2, G3, G4 and other additional treatment groups, $\mathrm{n}=2229$; CAL: $11.03 \%, \mathrm{p}<0.01)$. The odds ratio of the risk of developing CAL between the patients with IVIG resistance and responding patients was 6.32 .

Table 1 shows the incidence of CAL in each additional therapy group: $6.68 \%$ in $\mathrm{G} 2(\mathrm{n}=1108), 9.68 \%$ in G3 $(\mathrm{n}=93)$, and $22.22 \%$ in G4 $(\mathrm{n}=135)$. Statistical analysis revealed that the risk for development of CAL was significantly higher in G2, G3 and G4 than that in G1 ( $p<0.01, p<0.01$, and $\mathrm{p}<0.05$, respectively). Furthermore, G4 showed significantly higher incidence of CAL when compared with G2 and G3 ( $p<0.05$ and

Table 1. Incidence of CAL: comparison of four therapeutic groups.

\begin{tabular}{|c|c|c|c|c|}
\hline & ${ }^{*}$ G1 & ${ }^{*} \mathrm{G} 2$ & ${ }^{*}$ G3 & ${ }^{*} \mathrm{G} 4$ \\
\hline & No additional therapy & additional IVIG & additional steroids & additional IVIG plus steroids \\
\hline $\mathrm{N}$ & 9281 & 1108 & 93 & 135 \\
\hline Dilatation (\%) & 1.44 & $3.70^{@}$ & 2.15 & $5.93^{@}$ \\
\hline Aneurysm (\%) & 0.40 & $2.35^{@}$ & $3.23^{@}$ & $12.59^{@ \# \$}$ \\
\hline Giant aneurysm (\%) & 0.03 & $0.63^{@}$ & $4.30^{@ \#}$ & $3.70^{@ \#}$ \\
\hline Total CAL (\%) & 1.87 & $6.68^{@}$ & $9.68^{@}$ & $22.22^{@ \# \$}$ \\
\hline
\end{tabular}

${ }^{*} \mathrm{G} 1$, children responded to initial IVIG; G2, IVIG resistant patients receiving an additional IVIG; G3, IVIG resistant patients receiving an additional steroids; G4, IVIG resistant patients receiving an additional IVIG plus steroids; ${ }^{@}$ significant difference compared to G1 ( $\left.<0.01\right)$, ${ }^{\#}$ significant differ-

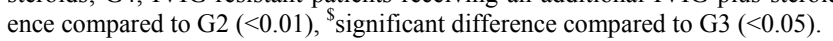


$\mathrm{p}<0.05$, respectively).

In regard to the type of CAL, the incidences of AN and GA were significantly higher in G2, G3, and G4 compared to that in G1 as well, whereas that of Dil was significantly higher only in G2 and G4 than that in G1. GA was more frequently seen in G3 and G4 consisting of the patients receiving steroids than in G1 and G2 consisting of the patients not receiving steroids (G1: $0.03 \%$, G2: $0.63 \%$, G3: 4.3\%, G4: 3.7\%).

\section{DISCUSSION}

The vast majority of children with respond promptly to treatment, which consists of an infusion of IVIG $(2 \mathrm{~g} / \mathrm{kg}$, administered over 12 hours) and high dose aspirin (30 $100 \mathrm{mg} / \mathrm{kg} / \mathrm{day}$ in three or four divided doses). Randomized, prospective clinical trials in Japan and US in the 1980s established that IVIG was an effective and safe treatment, which reduces the rate of CAL [1-3]. A proportion of patients (10\% to $20 \%$ ), however, fail to become afebrile despite treatment with IVIG and aspirin [3-8]. For those who did not respond to initial IVIG, most of the clinicians would attempt a repeated dose of IVIG at $2 \mathrm{~g} / \mathrm{kg}$ to achieve resolution of the inflammatory process with unsatisfactory results $[4,7,8]$.

Thus, an additional therapy for IVIG resistant KD has not been established and several treatment strategies other than secondary IVIG [14] have been explored as following: plasma exchange [12], methyl-prednisolone pulse therapy [10], ulinastatin [11], cyclophosphamide plus steroids [8], cyclosporin A plus steroids [15], methotrexate plus steroids [16] and infliximab [17,18].

In this study, we tried to clarify the efficacy and the risk of an additional therapy by IVIG and/or steroids on the incidence of CAL, because they were the most commonly applied therapeutic strategies for IVIG resistant KD patients not only in Japan but also in other countries. As a result, an additional IVIG dose was the most frequently used second line therapy for initial IVIG resistant $\mathrm{KD}$ patient $(49.7 \%)$, and the incidence of CAL in this group was $6.68 \%$ (GA: $0.63 \%$ ). Steroids were used as second line therapy in some patients $(4.27 \%)$ and the incidence of CAL was $9.68 \%$ (GA: $4.3 \%$ ). Statistical analysis clearly demonstrates a significant difference in the risk for development of CAL between patients without additional therapy (G1) and those with additional therapy (G2, G3 and G4; $p<0.01, p<0.01$, and $p<0.05$, respectively). As to the type of CAL, it was noteworthy that the patients receiving steroids (G3 and G4) revealed the higher incidence of GA (G3: $4.3 \%$, G4: 3.7\%) than those not receiving steroids (G1: $0.03 \%, \mathrm{G} 2: 0.63 \%$ ). These results suggest that steroids might increase the risk for GA formation, which was reported previously [19].

Although the reason why steroids increase the risk for developing aneurysms is currently unknown, steroids may mask the serial inflammation in IVIG resistant KD patients, thus resulting in some necessary subsequent treatments being delayed [20].

There are, however, some limitations in our study. First, several selection bias, i.e., more severe cases are given priority in allocation to G3 (IVIG resistant patients receiving steroids) or G4 (IVIG resistant patients receiving an additional IVIG plus steroids), may lead to the high incidence of GA in these groups. Second, considerable number of children in G4 received an additional IVIG plus steroids not as the secondary but as the third line therapy: it can be easily speculated that they did not respond to the secondary therapy and that the risk for CAL in those patients is significantly higher than that of those not requiring the third line therapy. Thus, more severe cases might have been included in G4 and this possibly affects the high incidence of GA in this group. Even though significantly higher incidence of GA in G4 might be due to selection bias, the finding that higher incidence of GA in G3 consisting of the IVIG resistant patients receiving only steroids suggest the causal relationship between the steroids use and GA.

In conclusion, incidence of CAL was significantly higher in patients with IVIG resistance than those responding to initial IVIG and subsequent steroids therapy may increase the risk for GA in children with initial IVIG resistant $\mathrm{KD}$, although a selection bias might affect this result. We therefore recommend an additional IVIG dose in patients with IVIG resistant KD while waiting the results of further studies to confirm the possible deleterious effect of steroids on the risk of CAL in this disease.

\section{ACKNOWLEDGEMENTS}

This study was partly supported by the Mami Mizutani Foundation.

\section{REFERENCES}

[1] Furusho, K., Kamiya, T., Nakano, H., Kiyosawa, N., Shinomiya, K., Hayashidera, T., et al. (1984) High-dose intravenous gammaglobulin for Kawasaki disease. Lancet, 2, 1055-1058. doi:10.1016/S0140-6736(84)91504-6

[2] Newburger, J.W., Takahashi, M., et al. (1986) The treatment of Kawasaki syndrome with intravenous gamma globulin. The New England Journal of Medicine, 315, 341-347. doi:10.1056/NEJM198608073150601

[3] Newburger, J.W., Takahashi, M., Beiser, A.S., et al. (1991) A single intravenous infusion of gamma globulin as compared with four infusions in the treatment of acute Kawasaki syndrome. The New England Journal of Medicine, 324, 1633-1639. doi:10.1056/NEJM199106063242305

[4] Burns, J.C., Capparelli, E.V., Brown, J.A., Newburger, 
J.W. and Glode, M.P. (1988) Intravenous gamma-globulin treatment and retreatment in Kawasaki disease. Pediatric Infectious Disease Journal, 17, 1144-1148. doi:10.1097/00006454-199812000-00009

[5] Durongpisitkul, K., Soongswang, J., Laohaprasitiporn, D., Nana, A., Prachuabmoh, C. and Kangkagate, C. (2003) Immunoglobulin failure and retreatment in Kawasaki disease. Pediatric Cardiology, 24, 145-148. doi:10.1007/s00246-002-0216-2

[6] Egami, K., Muta, H., Ishii, M., et al. (2006) Prediction of resistance to intravenous immunoglobulin treatment in patients with Kawasaki disease. Journal of Pediatrics, 149, 237-240. doi:10.1016/j.jpeds.2006.03.050

[7] Han, R.K., Silverman, E.D., Newman, A. and McCrindle, B.W. (2000) Management and outcome of persistent or recurrent fever after initial intravenous gamma globulin therapy in acute Kawasaki disease. Archives of Pediatrics \& Adolescent Medicine, 154, 694-699.

[8] Wallace, C.A., French, J.W., Kahn, S.J. and Sherry, D.D. (2000) Initial intravenous gamma globulin treatment failure in Kawasaki disease. Pediatrics, 105, e78-e81. doi:10.1542/peds.105.6.e78

[9] Newburger, J.W., Takahashi, M., et al. (2004) Diagnosis, treatment, and long-term management of Kawasaki disease: A statement for health professionals from the committee on rheumatic fever, endocarditis and Kawasaki disease, council on cardiovascular disease in the young, American Heart Association. Circulation, 110, 2747-2771. doi:10.1161/01.CIR.0000145143.19711.78

[10] Wright, D.A., Newburger, J.W., Baker, A. and Sundel, R.P. (1996) Treatment of immune globulin-resistant Kawasaki disease with pulsed doses of corticosteroidss. Journal of Pediatrics, 128, 146-149. doi:10.1016/S0022-3476(96)70447-X

[11] Okada, M., Nakai, S., Kobayashi, Y., et al. (1997) Effects of intravenous gamma globulin and ulinastatin on patients with Kawasaki disease and predicted giant coronary artery aneurysms. Journal of The Japan Pediatric Society,
101, 1165-1170.

[12] Mori, M., Tomono, N. and Yokota, S. (1995) Coronary arteritis of Kawasaki disease unresponsive to high-dose intravenous gammaglobulin successfully treated with plasmapheresis. Nihon Rinsho, 18, 282-288. doi:10.2177/jsci.18.282

[13] Research Committee on Kawasaki Disease (1984) Report of subcommittee on standardization of diagnostic criteria and reporting of coronary artery lesions in Kawasaki disease. Ministry of Health and Welfare, Tokyo.

[14] Sundel, R.P., Burns, J.C., Baker, A., Beiser, A.S. and Newburger, J.W. (1993) Gamma globulin re-treatment in Kawasaki disease. Journal of Pediatrics, 123, 657-659. doi:10.1016/S0022-3476(05)80972-2

[15] Raman, V., Kim, J., Sharkey, A. and Chatila, T. (2001) Response of refractory Kawasaki disease to pulse steroids and cyclosporin A therapy. Pediatric Infectious Disease Journal, 20, 635-637. doi:10.1097/00006454-200106000-00022

[16] Lee, M.S., An, S.Y., Jang, G.C. and Kim, D.S. (2002) A case of intravenous immunoglobulin-resistant Kawasaki disease treated with methotrexate. Yonsei Medical Journal, 43, 527-532.

[17] Weiss, J.E., Eberhard, B.A., Chowdhury, D. and Gottlieb, B.S. (2004) Infliximab as a novel therapy for refractory Kawasaki disease. Journal of Rheumatology, 31, 808810.

[18] Burns, J.C., Mason, W.H., et al. (2005) Infliximab treatment for refractory Kawasaki syndrome. Journal of Pediatrics, 146, 662-667. doi:10.1016/j.jpeds.2004.12.022

[19] Kato, H., Koike, S. and Yokoyama, T. (1979) Kawasaki disease: Effect of treatment on coronary artery involvement. Pediatrics, 63, 175-179.

[20] Furukawa, T., Kishiro, M., et al. (2008) Effects of steroid pulse therapy on immunoglobulin-resistant Kawasaki disease. Archives of Disease in Childhood, 93, 142-146. doi:10.1136/adc.2007.126144 